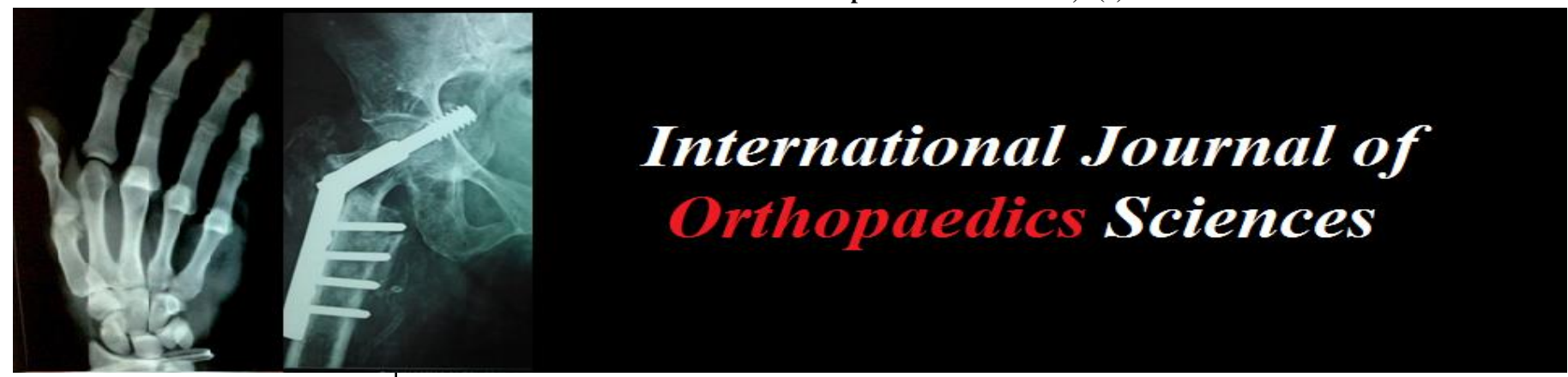

ISSN: $2395-1958$

IJOS 2019; 5(1): 70-74

(C) 2019 IJOS

www.orthopaper.com

Received: 09-11-2018

Accepted: 13-12-2018

Dr. Sharanprasad AH

Junior Resident, Department of Orthopedics, Shri B M Patil

Medical College Hospital and

Research Centre, Bijapur,

Karnataka, India

Dr. OB Pattanashetty

Professor and HOD of Orthopedics, Shri B M Patil

Medical College Hospital and Research Centre, Bijapur,

Karnataka, India

Dr. Ramanagouda Biradar Associate Professor of Orthopedics, Shri B M Patil Medical College Hospital and Research Centre, Bijapur, Karnataka, India
Correspondence

Dr. Sharanprasad AH

Junior Resident, Department of

Orthopedics, Shri B M Patil

Medical college hospital and

Research Centre, Bijapur,

Karnataka, India

\section{Prospective study in surgical management of femur neck fracture with hemiarthroplasty using modular bipolar prosthesis}

\author{
Dr. Sharanprasad AH, Dr. OB Pattanashetty and Dr. Ramanagouda \\ Biradar
}

DOI: $\underline{\text { https://doi.org/10.22271/ortho.2019.v5.i1b.16 }}$

\begin{abstract}
Background: The frequency of Femur Neck fracture, one of the most common traumatic injuries in the elderly increases continuously due to the ageing of population on the planet ${ }^{[1]}$. It has been predicted that by 2050 , the number of hip fractures would triple. Modular bipolar is an intermediate between the more type and total hip replacement, The primary goal of treatment of in the active elderly population is early restoration of premorbid walking ability and excellence of life

Materials and methods: A prospective, single blinded, randomized control trial with 33 cases, out of which 12 were males and 21 were females was conducted. The postoperative evaluation was done both clinically and radiologically. Out of the 33 cases, all patients were available for follow up till one year which was taken as a basic pre-requisite for inclusion in the study. At each follow up, patients were evaluated clinically using the Modified Harris Hip Score and radiologically with appropriate X-rays.

Results: The modified harris hip score where by the end of one year there is $81.9 \%$ of good functional outcome. During study period $3(9.7 \%)$ of the patients died due to medical conditions unrelated to surgical cause. the clinical outcome where in $27.2 \%$ had Excellent results, $45.5 \%$ had Good results and $12.1 \%$ had poor results. In our study we noticed that 4 of the patients were able to sit crossed leg and squat.

Conclusion: Hemiarthroplasty using Modular Bipolar prosthesis for fractures of the femoral neck provides freedom from pain, better range of movement and more rapid return to unassisted activity with an acceptable complication rate. The long term results using Modular bipolar prosthesis needs further study fora longer period in a larger sample with a direct comparison between the cemented versus uncemented groups. However, considering the good result achieved in the short term, it seems reasonable to use canal bone ratio in bipolar hemiarthroplasty as pre-operative template.
\end{abstract}

Keywords: Surgical management, femur neck fracture, hemiarthroplasty, modular bipolar prosthesis

\section{Introduction}

The frequency of Femur Neck fracture, one of the most common traumatic injuries in the elderly increases continuously due to the ageing of population on the planet ${ }^{[1]}$. It has been predicted that by 2050, the number of hip fractures would triple. At present leading cause of hospital admissions in elderly age group is Femur Neck fracture, because of increased longevity, osteoporosis and sedentary habits ${ }^{[2]}$.

Modular bipolar is an intermediate between the more type and total hip replacement, the Bipolar prosthesis which has mobile head element and has additional head surface to allow movement within the acetabulum. This led to reduced wear of acetabular surface and hence reduced occurrence of pain and acetabular protrusion because motion is present between the metal head and the polyethylene socket (Inner bearing) as well as between the metallic head and acetabulum (Outer bearing) ${ }^{[3]}$.

The primary goal of treatment of in the active elderly population is early restoration of premorbid walking ability and excellence of life. There are high chances and increased failure rates of using Internal fixation by dynamic hip screw or proximal femoral nail (avascular necrosis, nonunion, and repeat surgical procedures) ${ }^{[5,6]}$. 
Bipolar prosthesis is slowly replaced with the conventional unipolar prosthesis ${ }^{[8,9]}$. Bipolar prosthesis over monopolar prosthesis have higher percentage of satisfactory results, less post-operative pain, greater range of movements, more rapid return to noramal routine and reduced frequency of acetabular erosion ${ }^{[9,10]}$.

Primary Total Hip Replacement (THR) is being offered at many centres as a treatment option for these fractures with the superiority of prosthetic replacement over internal fixation being well established. Total hip arthroplasty is still not popular as a treatment modality for FEMUR NECK FRACTURE in our country because majority of the patients do well with hemiarthroplasty and also due to the high costs involved. It also has a higher occurrence of dislocations and higher morbidity associated with the procedure ${ }^{[11]}$.

The goal of the current study is to assess the functional and quality of life scores in patients treated with modular bipolar prosthesis for fracture of femoral neck.

\section{Materials and methods}

A prospective, single blinded, randomized control trial in the Department of Orthopaedics, Shri B M Patil Medical College Hospital and Research Centre, Vijayapura, Karnataka, India from october 2016-march 2018 was conducted after obtaining approval from College Ethics Committee. Written informed consent was undertaken before enrollment to the study.

\section{Inclusion Criteria}

- Intracapsular Femur Neck fractures

- Age of the patient $>60$ years

- Failed internal fixation

- Avascular necrosis of femoral head secondary to Femur Neck fracture

- Non union Femur Neck fracture

\section{Exclusion Criteria}

- Patient medically not fit for surgery

- Patient not willing for surgery

- Patient below age of 60 yrs

- Pathological hip fractures

- Patients with acetabular fractures

- Patients with hip arthritis

\section{Results}

Data was collected based on detailed patient evaluation with respect to history, clinical examination and radiological examination. The postoperative evaluation was done both clinically and radiologically. Out of the 33 cases, all patients were available for follow up till one year which was taken as a basic pre-requisite for inclusion in the study.

\section{Age Distribution}

Table 1: Shows the age distribution pattern of the patients. The average age was noted to be 67.9 years. The youngest patient in the study was 60 and oldest was 88 years. Mean age is 63.9 years.

\begin{tabular}{|c|c|c|}
\hline Age & Frequency & Percentage \% \\
\hline $60-69$ & 23 & 70.0 \\
\hline $70-79$ & 8 & 24.0 \\
\hline$>80$ & 2 & 06.0 \\
\hline
\end{tabular}

\section{Type of fracture}

Table 2: Shows that majority of study patients (66.7\%) had Gardens type III fracture and $18.2 \%$ patients had garden type IV fracture.

\begin{tabular}{|c|c|c|}
\hline Garden Classification & Frequency & Percentage \% \\
\hline Type I & 01 & 03.0 \\
\hline Type II & 04 & 12.1 \\
\hline Type III & 22 & 66.7 \\
\hline Type IV & 06 & 18.2 \\
\hline
\end{tabular}

\section{Modified harris hip score}

Table 3: Shows the modified harris hip score where by the end of one year there is $81.9 \%$ of good functional outcome. During study period $3(9.7 \%)$ of the patients died due to medical conditions unrelated to surgical cause.

\begin{tabular}{|c|c|c|c|c|}
\hline MHHS & $\mathbf{N}$ & Minimum & Maximum & Mean \\
\hline 6 Weeks & 33 & 30.00 & 80.00 & 70.00 \\
\hline 3 Month & 33 & 30.00 & 100.00 & 78.06 \\
\hline 6 Month & 31 & 70.00 & 100.00 & 80.86 \\
\hline 12 Month & 30 & 75.00 & 100.00 & 81.96 \\
\hline
\end{tabular}

\section{Clinical outcome}

Table 4: Shows the clinical outcome where in $27.2 \%$ had Excellent results, $45.5 \%$ had Good results and $12.1 \%$ had poor results. In our study we noticed that 4 of the patients were able to sit crossed leg and squat.

\begin{tabular}{|c|c|c|}
\hline & Frequency & Percentage \\
\hline Excellent & 9 & 27.2 \\
\hline Good & 15 & 45.5 \\
\hline Fair & 5 & 15.2 \\
\hline Poor & 4 & 12.1 \\
\hline Total & 33 & 100.0 \\
\hline
\end{tabular}
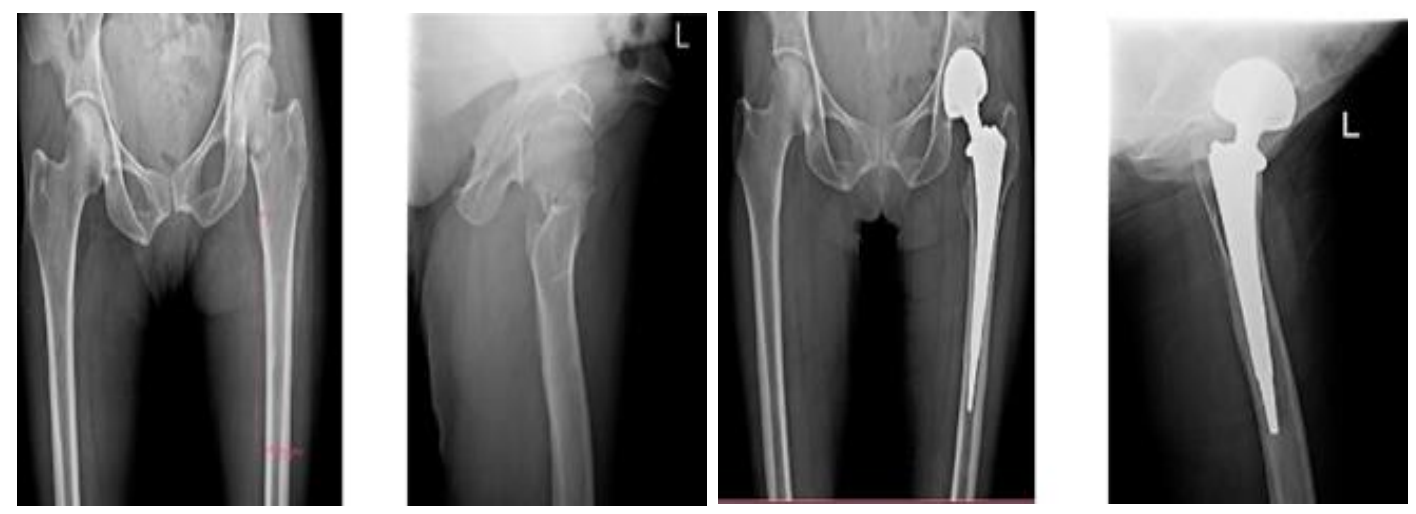

Image 1: pre and post operative X-ray of left neck of femur fracture treated with modular bipolar 

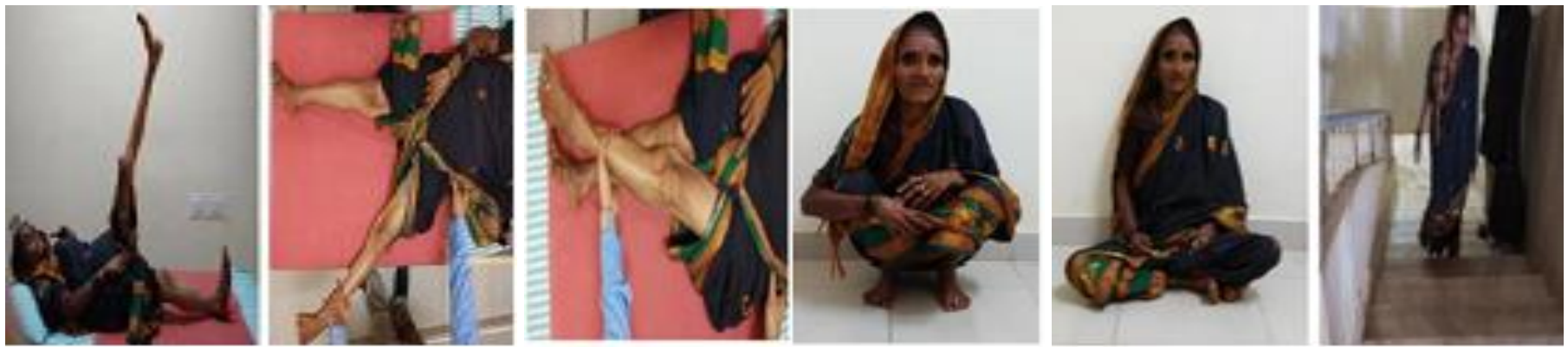

Clinical photograph
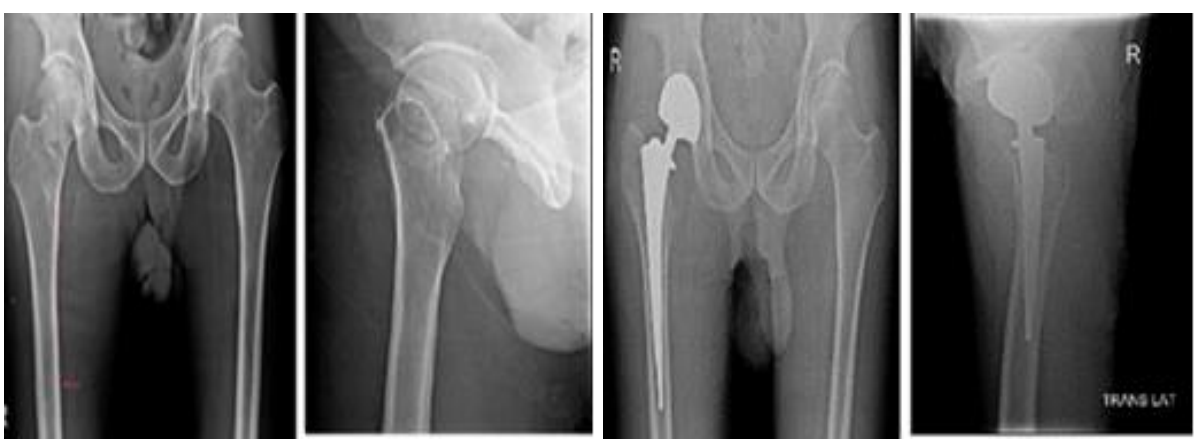

Image 2: Pre and post operative X-ray of left neck of femur fracture treated with modular bipolar
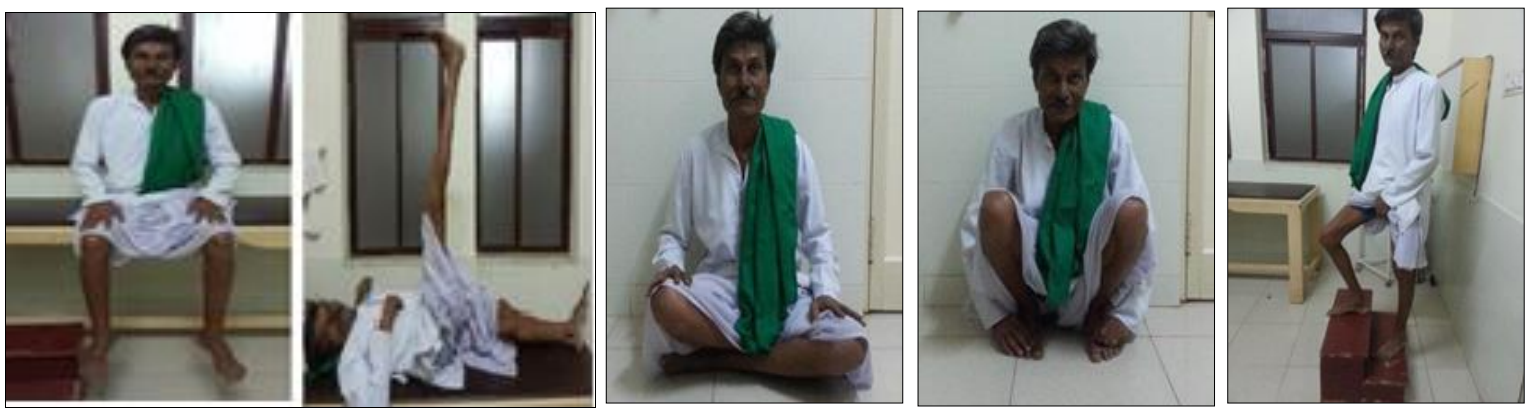

Clinical photograph

\section{Discussion}

Majority in our study patients $(75.8 \%)$ sustained the injury due to a trivial trauma like slipping. Most of such injuries can be classified as "indirect" trauma. Six patients (18.2\%) sustained the fracture secondary to a fall from a vehicle. A little more than one fourth of our study patients were brought to the hospital within seven days of sustaining the injury. 60.6 of the patients were brought to the hospital $7-14$ days of the injury and another $15.2 \%$ presented for treatment after 14 days.

Many of our study patients had a displaced fracture of the neck of femur. Majority of the patients $(66.7 \%)$ had Garden's type III and $18.2 \%$ had Type IV fracture. The anatomical type of fracture and the displacement are not considered when choosing hemiarthroplasty for management of fracture neck femur. This decision is based on the age of the and patient and time since injury ${ }^{[1]}$, Hypertension was found to be the most common co-morbidity seen in $18.2 \%$ of the study patients. Another $12.1 \%$ patients had only DM and both HTN and DM.

All the study patients were taken up for the surgical procedure between the 3rd and 30th day after the trauma, the average delay to surgery being 12 days. Delay in taking up for surgery was usually for optimizing the medical condition of the patient. All cases were performed on an elective basis and were scheduled as the first surgery in the morning.

All the surgeries were performed under spinal or epidural anaesthesia after a thorough pre anaesthetic evaluation and preparation. The choice of the type of anaesthesia was as per the anaesthetist's discretion.

All patients were operated after being put into lateral decubitus position by the posterior approach of Moore. The posterior approach was preferred because of the familiarity of most of the surgeons at our institution with the approach. Though the dislocation rate is reported to be more with the posterior approach, none of our study patients had a postoperative dislocation of the prosthesis ${ }^{[12]}$. This was because meticulous attention was given to insertion of prosthesis in 15-20 degrees anteversion, valgus positioning, suturing the posterior capsule and the short external rotators and keeping the limb in slight abduction after the procedure. Patients were also explained in the immediate pre-operative period about the risk of dislocation with excessive flexion or adduction and internal rotation of the hip. They were advised not to squat or sit cross leg, though 4 of our patients demonstrated squatting and sitting cross leg on follow up.

In 10 patients $43 \mathrm{~mm}$ prostheses were used. Cemented stems were used in 29 cases and Uncemented in 4 cases. The advantage was that the rasp corresponded to the exact length and width of the prosthesis which prevented any additional broaching of the canal and subsequent loose seating of the prosthesis or fractures of the femur. The medullary canal was washed with normal saline. Cement was prepared and finger packing into the canal was done. The prosthesis was then 
inserted and hammered in carefully maintaining correct anteversion.

Most of our study patients were mobilized in bed on day one of surgery and with weight bearing as tolerated using a walker, within the 72 hours postoperative period. Delay if at all was due to medical reasons.

Superficial infection in the form of a wound dehiscence was seen in five patient $(15.2 \%)$ who were a diabetic or hypertension and diabetic. Patient was with adequate control of the diabetic status and appropriate intravenous antibiotics based on pus culture-sensitivity results. One patient had a DVT post-operatively, confirmed by colour Doppler. This was managed with low-molecular weight heparin (Enoxaparin $40 \mathrm{mg}$ twice daily subcutaneously) for 5 days followed by oral anticoagulants (Warfarin $5 \mathrm{mg}$, titrated as per PT-INR values)

The minimum duration of hospital stay amongst the study patients was 12 days and maximum duration was 25 days with the average being 20 days, because patient required optimization of the co morbid conditions. Average hospital stay of 18 days reported by Han et al. ${ }^{[13]}$ In the series by Emery et al, the cemented group had an average stay of 21.8 days whereas it was 19.5 days in the uncemented group. Parker et al. ${ }^{[14]}$ found that hospital stay was mean 4 days shorter in those treated with a cemented prosthesis.

In our study post-operative follow up 1 patients had loosening of implant which resulted in anterior thigh pain. There were no late postoperative complications like dislocation, erosion, subsidence, protrusion acetabuli or periprosthetic fracture. We are unable to comment upon long term acetabular erosion due to relative short follow up.

All patients were followed up regularly at $6 \mathrm{wks}, 3$ months, 6 months, and one year. Only the patients who completed a minimum one year follow-up were included in the final analysis. The maximum follow up was for one year ten months and minimum of one year. The Modified Harris Hip Scores were recorded at each follow-up visit.

As Indian population needs to squat and sit cross leg for their daily living. In our study, we have used the Modified Harris Hip Score for the evaluation of the study subjects which includes squatting and sitting crossleg ${ }^{[14]}$. In our study using Modified harris hip score as evaluated at one year follow-up averaged 81.96 with the maximum score being 100and the minimum score being 75 . Overall, 6 patients $(19.4 \%)$ achieved Excellent result, 22 patients $(71 \%)$ achieved Good result, and 3 patients $(9.7 \%)$ achieved poor result. Overall, $77.27 \%$ of the patients achieved an excellent or good result.

Our results seem to suggest that uncemented stem of Modular Bipolar prosthesis gives a better functional, lesser pain and improved gait function outcome by providing better primary anchorage of the prosthesis. This is especially important in the osteoporotic femur with weak calcar. A good stable fit allows early mobilization of patients. Though it is against the common practice of inserting the AMP without cement, this might need a change, because this prosthesis in now used in the very elderly patients with limited demands and mobility, due to better outcome with bipolar hemiarthroplasty in physiologically younger patients. Due to the limited life expectancy of these patients, revision is not a major issue. Lesser pain, better mobility and early mobilization is what is important and these goals are better achieved when the Uncemented stem of Modular Bipolar prosthesis in place.

\section{Conclusion}

Hemiarthroplasty using Modular Bipolar prosthesis for fractures of the femoral neck provides freedom from pain, better range of movement and more rapid return to unassisted activity with an acceptable complication rate. Though conventionally done in an uncemented fashion, providing a good primary anchorage, especially in the osteoporotic femur is of paramount importance. The canal bone ratio is helpful in predicting the use of prosthesis i.e. uncemented or cemented preoperatively. This step helps reduce thigh pain and achieve better functional outcomes in terms of better mobility and lesser use of walking aids. The end functional results also depend on the associated co-morbidity and optimum postoperative rehabilitation. Throughout the purview of the present study, our experience with uncemented Modular bipolar prosthesis has been better. The long term results using Modular bipolar prosthesis needs further study fora longer period in a larger sample with a direct comparison between the cemented versus uncemented groups. However, considering the good result achieved in the short term, it seems reasonable to use canal bone ratio in bipolar hemiarthroplasty as pre-operative template.

\section{References}

1. Filipov O. Epidemiology and social burden of the Femur Neck Fracture. J of IMAB. 2014; 20(4):516-518.

2. Robert Döring, Thorsten Jentzsch, Max Scheyerer J, William Pfäffli, Clément Werner ML. The value of modular hemiarthroplasty for unstable Femur Neck Fracture in elderly patients with coxarthrosis. BMC musculoskeletal disorders. 2016; 17:223.

3. Hanu Tej Adapureddi, Kamareddy SB, Anand Kumar, Sri Krishna Paturi, Sandeep Anne, Jaya Prakash Reddy. Prospective Study of Management of Femur Neck Fracture by Hemiarthroplasty with Cemented Bipolar. Journal of Evolution of Medical and Dental Sciences. 2015; 4(98):16309-16314.

4. Bhandari M, Devereaux PJ, Swiontowski MF, Tornetta P, Obremskey $\mathrm{W}$, Koval $\mathrm{KJ}$ et al. Internal fixation compared with arthroplasty for displaced fractures of the femoral neck. J Bone Joint Surg Am. 2003; 85:16731681.

5. Ioro R, Schwartz B, Macaulay W, Teeney SM, Healey WL, York S. Surgical treatment of displaced Femur Neck Fracture in the elderly: A survey of the American Association of Hip and Knee Surgeons. J Arthroplasty. 2006; 21(8):1124-1133.

6. Marya S, Thukral R, Hasan R, Tripathi M. Cementless bipolar hemiarthroplasty in Femur Neck Fracture in elderly. Indian J Orthop. 2011; 45:236-42.

7. Swiontowski MF. Intracapsular fractures of the hip. J Bone Joint Surg Am. 1994; 76:129-138.

8. Zofka P. Bipolar hip hemiarthroplasty. Acta ChirOrthop Traumatol Cech. 2007; 74(2):99-104.

9. Malhotra R, Arya R, Bhan S. Bipolar hemiarthroplasty in Femur Neck Fracture. Archives of Orthopaedic and Trauma Surgery. 1995; 114(2):79-82.

10. Sud A, Sood LK. Bipolar hip replacement for displaced Femur Neck Fracture in elderly patients. Indian Journal of Orthopaedics. 1998; 32:270-271.

11. Maini PS, Talwar N, Nijhawan VK, Dhawan M. Results of cemented bipolar hemiarthroplasty for fracture of the femoral neck - 10 year study. Indian Journal of Orthopaedics. 2006; 40(3):154-156.

12. Haidukewych GJ, Israel TA, Berry DJ. Long term survivorship of cemented bipolar hemiarthroplasty for fracture of the femoral neck. Clin Orthop. 
13. Anil Rai K, Rakesh Agarwal, Saurabh Singh, Ratnav Ratan. The BHU bicentric bipolar prosthesis in fracture neck femur in active elderly. Journal of Trauma Management \& Outcomes. 2008; 2:7.

14. Han SK, Kim YS, Kang SH. Treatment of Femur Neck Fracture with bipolar hemiarthroplasty using a modified minimally invasive posterior approach in patients with neurological disorders. Orthopaedics. 2012; 35(5):e63540. 